\title{
DISCUTINDO CITY INFORMATION MODELING (CIM) E CONCEITOS CORRELATOS
}

\section{Discussing City Information Modeling (CIM) and related} concepts

\section{Arivaldo Leão de Amorim}

RESUMO Este artigo discute o paradigma City Information Modeling (CIM) a partir de uma abordagem ampla, considerando um viés teórico e conceitual deste e de outros ternos relacionados. O CIM tem sido apontado por alguns autores como uma extensão do conceito de BIM para o espaço urbano. Contudo, face às características da cidade em relação à edificação isolada e à profusão de conceitos ora em uso, algumas questões precisam ser aprofundadas. Esses conceitos estão intimamente relacionados, apresentando algum tipo de sobreposição (e talvez, por esse motivo, sejam confundidos). Assim, pretende-se ampliar a discussão sobre esses conceitos, contribuindo para a utilização destes e avançando no estabelecimento de uma conceituação e de terminologias que facilitem o desenvolvimento das aplicações e a adoção das tecnologias nas práticas relativas ao planejamento, à gestão e ao monitoramento da cidade. Não há intenção alguma de se esgotar o tema, seja pela vastidão das questões envolvidas ou pelo reduzido espaço para a discussão destas, mas principalmente, porque estas questões estão "em aberto" e são objetos de discussões e controvérsias.

PALAVRAS-CHAVE: City Information Modeling (CIM), Building Information Modeling (BIM), City Geography Markup Language (CityGML), 3D Geographic Information System (3D GIS), SmartCity.

ABSTRACT This paper discusses the City Information Modeling (CIM) paradigm from a wide approach, considering the theoretical and conceptual bias on those and other related terms. The CIM has been pointed by some authors as an extension of the BIM concept for the urban space. However, considering the characteristics of the city comparing to an isolated building and the profusion of concepts in use, some issues need a more deep discussion. These concepts are closely related and have some overlap (that is why sometimes they are misunderstood). Thus, we intended to broaden the discussion to clarify the concept and terminology, and contribute to their use, in addition to improving the development of applications and technologies for planning, management and monitoring the cities. We have no intention of exhausting the theme, due the vastness of issues involved and the reduced space for discussion, but mainly because these issues are still "open", promoting discussion and controversy.

KEYWORDS: City Information Modeling (CIM), Building Information Modeling (BIM), City Geography Markup Language (CityGML), 3D Geographic Information System (3D GIS), SmartCity.

\section{How to cite this article:}

AMORIM, A. L. Discutindo City Information Modeling (CIM) e conceitos correlatos.

Gestão e Tecnologia de Projetos, São Paulo, v. 10, n. 2, p. 87-99, jul./dez. 2015

http://dx.doi.org/10.11606/gtp.v10i2.103163

Fonte de financiamento: $\mathrm{CNPq}$ - Conselho Naciona de Desenvolvimento Científico e Tecnológico Conflito de interesse: Declaram não haver Submetido em: 1 set. 2015 Aceito em: 1 set. 2015 


\section{CITY INFORMATION MODELING}

Com a crescente complexidade dos Sistemas do Mundo Real (SMR), a contínua evolução das tecnologias digitais e a consequente e acelerada integração das várias disciplinas da área de Arquitetura, Engenharia, Construção e Operação (AECO), nada mais natural e esperado que o surgimento de novos paradigmas. Neste artigo discute-se o City Information Modeling, ou simplesmente CIM, como está sendo difundido.

Mas, o que é City Information Modeling?

Segundo Hisham (2010), “[...] mais recentemente foi cunhado o um novo termo CIM ou modelo de Informações sobre a cidade, o qual visa transformar o modo como os planejadores urbanos lidam com os seus planos a semelhança dos arquitetos."1 [tradução nossa].

Embora à primeira vista esta afirmativa possa parecer interessante e fazer sentido, o trabalho realizado pelo arquiteto projetista é bastante diferente do que é feito pelo arquiteto planejador urbano. Isto acontece não apenas por tratarem de objetos diferentes, a edificação e a cidade, mas principalmente, pela natureza intrínseca de suas atividades. Por consequência, utilizam ferramentas de características bastante diversas.

Enquanto na projetação da edificação (atividade cujo efeito se dará em curto prazo), são detalhados ao extremo os diversos aspectos do objeto em foco, no planejamento urbano (a atividade cujas ações são propostas para acontecerem e produzirem efeitos em horizontes de curto, médio e longo prazo, de 5 a 20 anos), são fixados as diretrizes gerais de zoneamento, os parâmetros e os indicadores urbanos desejados, mas num grau de generalizão e abstração incompatível com qualquer projeto executivo.

Outro aspecto relevante é a grande liberdade que os projetistas possuem para trabalhar com formas e materiais, enquanto os planejadores estão submetidos a um grande número de restrições, e, normalmente, trabalhar as formas e os materiais não faz parte do seu escopo do trabalho, que está focado em coletar dados do mundo real, analisá-los e propor cenários futuros.

Por estas e outras razões, considera-se que a afirmativa de Hisham (2010) é muito simplista, não contribuindo para o entendimento da ideia de CIM em toda a sua extensão e complexidade.

Quanto à origem do termo City Information Modeling (CIM), Gil et al. (2010) sugerem que este teria sido forjado por Lachim Khemlami, editor e fundador da publicação on-line AECbytes. Desde a criação da publicação em 2003, Khemlami vem pesquisando, analisando e revisando produtos e serviços baseados em Tecnologias de Informação e Comunicação (TIC) para a indústria da construção civil.

Em seus estudos, Gil, Almeida e Duarte (2011) apontam que:

“O CIM poderá estender o uso dos Sistemas de Informações Geográficas (SIG) no planejamento urbano como ferramentas de apoio à decisão (WEBSTER, 1993; BATTY et al., 1998), através da integração com o Projeto Assistido por Computador (CAD), constituindo uma ferramenta de apoio ao projeto” (DAVE e SCHMITT, 1994; MAGUIRE, 2003, tradução nossa) ${ }^{2}$.

Assim, os autores entendem o CIM como equivalente ao conceito de Building Information Modeling (BIM) para a projetação urbana (GIL; ALMEIDA; DUARTE, 2011, p. 143).

Segundo os mesmos autores,

"[...] more recently a new term was coined, CIM or the City Information Model, which aims to transform the urban planners way of handling their plans just like architects."

"The CIM would extend the use of Geographic Information Systems (GIS) in urban planning as decision support tools (Webster, 1993; Batty et al., 1998) through the integration with Computer Aided Design (CAD), to become a design support tool (Dave and Schmitt, 1994; Maguire, 2003).” 
O projeto de pesquisa "City Induction" foi focado no desenvolvimento de um sistema baseado em um método de projetação urbano que integra as etapas de formulação, geração e avaliação de projetos urbanos apoiados por uma plataforma de software CAD e GIS (Ibidem, tradução nossa) ${ }^{3}$.

Portanto, esse projeto reduz o conceito do CIM ao âmbito da projetação urbana, não levando o conceito até as últimas consequências, tornando-se, assim, um paradigma capaz de abranger todo o "ciclo de vida" da cidade ou dos sistemas urbanos.

No mesmo sentido, Stojanovski (2013) estabelece que:

“CIM é uma analogia ao BIM em urbanismo. É um sistema de elementos urbanos representados por símbolos em um espaço 2D e dentro de um espaço 3D. Ele também é concebido como expansão 3D do GIS (SI3D ou Sistema de Informação 3D) enriquecido com vistas em vários níveis e múltiplas escalas, caixa de ferramentas de projeto e inventário de elementos 3D com seus relacionamentos” (STOJANOVSKI, 2013, p. 4, tradução nossa) ${ }^{4}$.

Muito embora Stojanovski (2013) conceitue desta maneira, sua visão explicitada no artigo "City Information Modeling (CIM) and Urbanism: Blocks, Connections, Territories, People and Situations" aproxima o CIM mais de um recurso para análise das complexas relações urbanas do que de um conjunto de ferramentas para a projeção e a gestão urbanas. Paradoxalmente, descreve uma situação muito mais próxima do Geographic Information Systems, que ele considera limitada para as aplicações urbanas, do que de uma visão de Building Information Modeling, considerada por ele como mais adequada para o enfrentamento das questões por ele apresentadas.

Contudo, face às características da cidade em relação à edificação isolada, cabe explicitar quais dimensões ou aspectos da cidade se pretende considerar nesse tipo de modelagem. Se, por um lado, a ideia ou conceito de BIM já é por si só bastante amplo e complexo, por outro, a ideia ou o conceito de CIM envolve uma complexidade e problemas muito maiores.

Enfim, a presente discussão aborda a profusão de conceitos já cunhados e em uso, mostrando que eles estão intimamente relacionados e apresentam algum tipo de sobreposição. Esses conceitos são por vezes confundidos, a exemplo de SmartCity; 3D Geographic Information Systems (3D GIS), City Geography Markup Language (CityGML) e os clássicos conceitos de planejamento, gestão e monitoramento urbanos, só para ficar entre os mais difundidos.

Entende-se que para o estabelecimento e a consolidação de um "paradigma CIM", independentemente das questões de ordem tecnológicas, existem algumas questões conceituais que precisam ser discutidas e aprofundadas pela comunidade envolvida na busca de uma definição consensual.

Assim, pretende-se fomentar a discussão sobre esses e outros conceitos relacionados contribuindo para sua difusão. Isto se desdobra no desenvolvimento de aplicações e na adoção de novas ferramentas nas práticas relativas ao planejamento, à projeção, à construção, à gestão (operação e manutenção) e ao monitoramento das cidades, como deve ser o escopo do CIM.

\section{ESTABELECENDO UM CONTEXTO PARA A DISCUSSÃO}

Buscando contribuir para uma maior e melhor explicitação do contexto, são aqui apresentados e discutidos alguns conceitos que constituem o

\footnotetext{
"The City Induction research project has been focusing on the development of such a system, based on an urban design method that integrates the stages of formulation, generation and evaluation of urban designs supported by a CAD/GIS software platform."

"CIM is a BIM analogy in urbanism. It is a system of urban elements represented by symbols in $2 D$ space and the $3 D$ spaces within. It is also conceived as $3 D$ expansion of GIS (3DIS or $3 D$ information system) enriched with multilevel and multiscale views, designer toolbox and inventory of $3 D$ elements with their relationship."
} 
background do City Information Modeling, que vão desde o conceito de SmartCity, passando pelas funções da administração urbana e, finalmente, chegando às tecnologias e ferramentas que dão suporte aos diversos sistemas de automação e viabilizam as aplicações.

\section{SmartCity}

O conceito de SmartCity, SmarterCity ou "cidade inteligente" é sutil e controverso. Vários outros termos têm sido empregados para expressar conceitos mais ou menos semelhantes, como cyberville, digital city, eletroniccommunities, flexicity, information city, intelligent city, knowledgebased city, MESH city, telecity, teletopia, ubiquitous city, wirecity dentre outros (WIKIPEDIA, 2014).

Embora esses conceitos possam diferir um pouco segundo os vários autores, a principal ideia por trás deles consiste no uso intensivo e extensivo das Tecnologias de Informação e Comunicação (TIC), empregando todo o potencial destas visando aperfeiçoar o desempenho dos serviços urbanos, de modo a suportar o desenvolvimento econômico, social e cultural; contribuir para o estabelecimento das chamadas “indústrias criativas”; propiciar bem estar para todos os usuários da cidade e ainda assegurar a participação eficaz dos cidadãos em todas as questões que lhe dizem respeito ${ }^{5}$.

Por meio do uso disseminado das tecnologias de informação e comunicação, espera-se que uma "cidade inteligente" responda mais rapidamente aos desafios globais e às suas próprias demandas, sendo capaz de aprender, inovar e se adaptar de forma eficaz às mudanças de contexto.

O crescente interesse por este tipo de abordagem tem sido motivado pelos grandes desafios do final do século passado, como a globalização acelerada, o crescimento e a reestruturação econômica mundial, o envelhecimento da população nos países mais desenvolvidos, o crescimento da indústria do entretenimento e do varejo on-line, as pressões sobre as finanças públicas, as mudanças climáticas, dentre outros fatores.

Selada e Silva (2013) apontam um estudo da União Europeia realizado pela Technische Universität Wien, pela University of Ljubljana e pela Delft University of Technology, que considera a existência de seis "indicadores de inteligência” para uma SmartCity, a saber: economia, governança, mobilidade, pessoas, ambiente e modo de vida.

De forma sintética, estes indicadores representam a competitividade econômica das cidades considerando aspectos relacionados à inovação e ao empreendedorismo. A governança diz respeito a questões relativas à cidadania, como a participação e a qualidade dos serviços e da administração pública nos seus vários níveis. A mobilidade está relacionada aos sistemas de transportes internos e externos, e à disponibilidade e qualidade das redes de informação e comunicação. O indicador "pessoas" considera o grau de qualificação dos recursos humanos, a abertura e o nível das interações sociais. $\mathrm{O}$ ambiente é definido pela atratividade das condições naturais, proteção ambiental e gestão dos recursos. Finalmente, o indicador modo de vida integra diversas questões ligadas à qualidade de vida, como habitação, saúde, segurança, cultura e turismo.

Concluindo, na visão de Lee e Hancock (2012) existem 143 projetos de SmartCities distribuídos pelo mundo, sendo 47 na Europa, 40 na Ásia, 35 na América do Norte, 11 na América do Sul e 10 na África e no Médio Oriente. Esses autores afirmam ainda que, enquanto na Europa e na América do Norte tem prevalecido projetos de renovação urbana (como o Amsterdam SmartCity na Holanda e o SmartSantander na Espanha), na Ásia e no Oriente Médio as iniciativas apontam para o planejamento e a construção

Outros conceitos relacionados são: e-government, e-participation, e-education e outros. Entretanto, o aprofundamento destes temas foge ao escopo deste trabalho. 
de cidades inteiras a partir do zero, como Masdar nos Emirados Árabes Unidos e Songdo na Coreia do Sul.

\section{O planejamento, a gestão e o monitoramento urbano}

Diferentemente de uma edificação, uma cidade raramente é planejada, projetada, construída, ocupada e usada num curto prazo. Brasília e outras poucas cidades são raros exemplos disto. As cidades estão em continua transformação, aumentando a sua complexidade e com demandas sempre crescentes, seja pelo novo, seja pela melhoria daquilo já existente.

Crescendo e adensando a população, expandindo o perímetro urbano, ocupando novas áreas e verticalizando as já construídas, transformando o uso e a ocupação do solo, e ampliando sua impermeabilização, ou ainda por meio do aperfeiçoamento ou da implantação novos sistemas de infraestrutura, as cidades vão crescendo, transformando-se e reinventandose num ciclo de vida dinâmico e complexo. Este ciclo não é igual para toda a cidade ou mesmo para as suas partes ou subsistemas. Assim, com essa dinâmica, as cidades raramente morrem, elas são sempre renovadas ${ }^{6}$.

Nesse sentido, os tradicionais recursos de enfrentamento das questões urbanas pelas administrações públicas compreendem o planejamento, a gestão e o monitoramento urbanos. Enquanto o planejamento foca na elaboração de estudos, planos, projetos, legislação etc., fica no âmbito da gestão executar o planejamento e as demais ações, ou seja, a construção da infraestrutura e das edificações com a implantação, operação e manutenção de todos os sistemas e serviços públicos. Finalmente, cabe ao monitoramento as funções de controle, de fiscalização e de verificação da qualidade dos serviços públicos ofertados à população e o impacto disto sobre o meio ambiente, de modo a realimentar os processos de planejamento e gestão num contínuo aperfeiçoamento.

Até então, os recursos de planejamento, gestão e monitoramento sempre deram conta, com maior ou menor eficácia, das necessidades urbanas e das transformações delas decorrentes. Entretanto, o contexto que agora se apresenta é o das megacidades, da superpopulação e da sustentabilidade frente à escassez de solo, água e energia, dentre outros recursos naturais, aliado à demanda crescente por novos serviços, a expansão dos padrões de consumo, a incorporação de parcelas das populações que antes não eram atendidas ou estavam marginalizadas, o combate à poluição, as mudanças climáticas e a proteção ao meio ambiente. Somam-se a isso os crescentes custos econômicos e financeiros para o provimento de todas as ações e os serviços necessários para fazer frente a esses desafios. Para atender a todas estas questões, novas ferramentas e estratégias têm sido propostas e desenvolvidas.

\section{D Geographic Information Systems (3D GIS)}

Geographic Information Systems (GIS) ou Sistemas de Informações Geográficas (SIG) podem ser entendidos como bancos de dados espaciais, constituído por um mapa base contendo as feições de interesse da aplicação e seus atributos.

Mais recentemente tem sido comum a representação da superfície da terra por Modelos Digitais de Terreno (MDT) que cumprem o papel dos mapas e objetos geográficos (vias, edificações, acidentes naturais) ou feições a serem representados por objetos 3D digitais. Os sistemas de informações geográficas dotados dessas características têm sido chamados de 3D_GIS, 
para diferenciá-los dos sistemas baseados em representações 2D, ou seja, os mapas digitais tradicionais.

O mapa base, as feições e seus atributos semânticos, juntos de todas outras informações associadas a eles, constituem a base de dados do SIG. A partir dos dados armazenados é possível elaborar consultas, análises e a visualização de informações, além de realizar simulações diversas e a formulação de cenários alternativos. Como resultado das operações efetuadas sobre a base de dados é possível gerar produtos como mapas, relatórios, tabelas e gráficos que documentam os estudos, as análises, as projeções, as propostas e os cenários (BURROUGH; McDONNELL, 1998; GOODCHILD, 2006).

No contexto urbano, esta tecnologia tem sido usada principalmente no desenvolvimento de aplicações voltadas às questões de planejamento, de gestão e de monitoramento, como a operação dos vários sistemas e serviços, a mitigação de desastres e, de forma ainda incipiente, em aplicações no âmbito do patrimônio histórico e cultural. O desenvolvimento dos 3D_GIS para a representação de cidades e suas aplicações tem sido chamado de Urban Information Modeling.

Os SIG são especificados, projetados e implantados para representar um ou mais sistemas do mundo real. Os SIG podem ser desenvolvidos para suportar ações de planejamento, gestão (operação e manutenção) e monitoramento de todos os sistemas urbanos, a exemplo de segurança e políticas públicas, e sistemas de infraestrutura, como telecomunicações, transportes públicos e saneamento, ou sistemas sociais como educação e saúde, dentre outros.

Os SIG voltados para a operação e a manutenção de redes e dos sistemas de infraestrutura são denominados de Computer Aided Facilities Management (CAFM). Um recurso poderoso, à disposição destes sistemas e que os potencializa ainda mais, é a possiblidade de receber informações online por meio de sistemas de telemetria, permitindo a alimentação dinâmica da base de dados e o acompanhamento contínuo do desempenho do sistema do mundo real representado na aplicação.

Enquanto Burrough (1998) considera os SIG como um poderoso conjunto de ferramentas para coletar, armazenar, recuperar, transformar e visualizar dados, autores como Cowen (1988) estabelecem que os SIG podem ser melhor definidos como sistemas de apoio à decisão, envolvendo a integração de dados georreferenciados em larga escala para os mais diversos tipos de aplicações.

De qualquer forma, embora os SIG apresentem valiosos recursos para o planejamento, gestão e monitoramento das cidades, eles estão muito longe de serem ferramentas de projeto, no contexto da Arquitetura, da Engenharia ou do Urbanismo.

\section{City Geography Markup Language}

O City Geography Markup Language ou CityGML, como é mais conhecido, constitui um padrão internacional e neutro para um modelo de dados e formato para intercâmbio de dados e informações geoespaciais que foi desenvolvido na Europa e está sendo usado em escala mundial para a representação tridimensional digital de cidades.

O modelo de dados proposto é capaz de representar todas as partes relevantes da cidade, com sua aparência, geometria, topologia e semântica, em formato numérico. O padrão foi estabelecido em 2008 pelo Open Geospatial Consortium (OGC) com o lançamento da versão CityGML 1.0. A versão mais recente, a 2.0, foi aprovada em 2012 incorporando significativas melhorias e constituindo um padrão de codificação aberto, não proprietário e abrangente para a representação das cidades.

O padrão contempla a representação de todos os objetos da cidade (CityObjects) desde o terreno (MDT), as edificações, as redes de transportes, 
os corpos d’água, o mobiliário urbano, as linhas de transmissão, a vegetação e outros elementos. Suas principais características são a representação visual baseada em cinco níveis de detalhes (Level of Detail-LOD) que variam do mais geral e esquemático (LOD 0) para o mais específico e detalhado (LOD 4); a definição de uma única estrutura geométrica e topológica para os objetos; o uso de mapeamento de texturas e o acesso livre e isento de custos (GRÖGER et al., 2012).

O CityGML é portanto um padrão para a representação tridimensional digital dos elementos físicos da cidade e suas relações, que visa a interoperabilidade dos sistemas e aplicações de natureza urbana. Assim, pode-se estabelecer que o CityGML é um padrão para a implementação dos 3D_GIS. Sendo considerado o núcleo do modelo de informações para os modelos tridimensionais numéricos de cidades (Computer City Models). São exemplos desse tipo de aplicação os modelos de Berlim, Colônia, Dresden, Munique e do Campus Norte do Karlsruhe Institute of Technology, dentre outros (GRÖGER et al., 2012).

Por fim, cabe observar que embora o padrão CityGML tenha sido pensado e desenvolvido inicialmente para a cidade, ou seja o urbano, os objetos representados possuem significância e abrangência regional, como os sistemas de transportes, as linhas de transmissão de energia, as adutoras, os rios, os canais, as bacias hidrográficas etc. Isto possibilita a extensão das aplicações para o município como um todo e mesmo para territórios mais vastos.

\section{CIM NA VISÃO DOS PROVEDORES DE TECNOLOGIA}

Como é possível observar, mesmo no plano teórico, a conceituação de City Information Modeling não é pacífica e uma definição consensual está longe de ser estabelecida. Além disso, no que se refere aos aspectos tecnológicos implementados em pacotes de ferramentas necessárias ao desenvolvimento das aplicações práticas, o quadro ainda é de indefinição ou de perplexidade.

No sentido de entender melhor o novo conceito e sua perspectiva de aplicação prática, procurou-se identificar a visão das softhouses sobre o tema, por meio de consultas aos websites e a documentos oficiais de três importantes representantes da indústria de software para AECO: Autodesk, Bentley e Esri.

\section{Autodesk}

Curiosamente, na busca realizada no website da Autodesk, fabricante da ferramenta CAD-BIM mais difundida, verificou-se que não existe um único white paper dedicado ao City Information Modeling (CIM), nem mesmo uma discussão ou menção explícita ao conceito.

Entretanto no white paper "BIM for Infrastructure: a vehicle for business transformation” (AUTODESK, 2012) a empresa expõe a sua visão e estratégia para a área de infraestrutura, o acrônimo CIM aparece com outro significado, ainda assim guardando alguma relação.

O documento de certa forma justifica a não adoção do termo City Information Modeling, mostrando que a Autodesk prefere apostar no conceito "BIM for infrastructure", quando explicita que:

Diferentes títulos e acrônimos foram testados para descrever uma abordagem baseada em modelos para projeto em engenharia civil, engenharia virtual e construção (VDC) e gerenciamento de informações civis (CIM), para citar dois. No entanto, fica claro que apesar da inicial resistência para o termo aplicado à engenharia 
civil e disciplinas relacionadas, o BIM para infraestrutura está se tornando corrente (AUTODESK, 2012, p. 18, tradução nossa) 7 .

As ferramentas que poderiam ser associadas a aplicações relativas aos temas urbanos e cidades são rotuladas como "Infrastructure Design Suite" ou "Civil Infrastructure", que compreendem vários pacotes de ferramentas para projetos diversos na área da engenharia civil, mas longe ainda de esgotarem todas as necessidades da "projetação urbana".

Embora muitas dessas ferramentas utilizem recursos de modelagem paramétrica e outros conceitos do momento, o seu principal formato de arquivo de dados é o DWG. No que pese este tenha sido e continue sendo um importante e eficiente formato de arquivo para o armazenamento de informações gráficas, é um formato pobre em semântica, bem distante daquilo que se entende como necessidade do BIM ou do conceito de interoperabilidade.

$\mathrm{Na}$ busca realizada não foram encontradas referências a uma futura incorporação do padrão IFC (Industry Foundation Classes) para o conjunto de ferramentas citadas ou mesmo a outro formato de arquivo proprietário, a exemplo do RVT, que venha a ser mais adequado para a aplicação integrada e seja dotada de riqueza semântica, como se entende ser o CIM.

Em outras palavras, a filosofia e as tecnologias contidas nessas ferramentas estão muito mais próximas do conceito de "CAD stand alone" (ou seja, da projetação por meio de disciplinas individualizadas) do que de uma abordagem integrada e colaborativa, dotada de recursos interoperáveis e com o foco no ciclo de vida do empreendimento, como é o caso do paradigma BIM.

\section{Bentley}

Num case study showcase divulgado pela Bentley (2011) denominado "City Information Modeling for Sustaining Cities - Lessons Learned from Advanced Users" são apresentadas três aplicações que envolvem a modelagem urbana desenvolvida com auxílio de produtos da empresa.

Os projetos citados compreendem o 3D City Model de Helsinki, iniciado em 1987, o 3D City Model de Montreal e o projeto Crossrail em Londres, iniciado em 2001, e que detalha a implantação de uma linha metroviária de $118 \mathrm{~km}$ de extensão, cortando a cidade. É importante destacar que, segundo a publicação, os modelos produzidos nessas aplicações são detalhados, precisos e destinados não apenas à visualização, mas, sobretudo, são importantes recursos usados no planejamento, na projetação e na gestão urbanas.

Na visão da própria Bentley:

Cada uma das equipes de projeto compartilha um objetivo comum: gerenciar complexos projetos de infraestrutura no ambiente urbano da maneira mais eficiente. As visões por trás das estratégias desenvolvidas por estes diferentes organizações se baseiam no mesmo princípio: uso de um modelo 3D inteligente para apoiar a colaboração em todo o ciclo de vida de ativos de infraestrutura, incluindo o planejamento, projeto, engenharia (sic), construção e o gerenciamento na fase de operação (BENTLEY, 2011, tradução nossa). ${ }^{8}$

Das informações coletadas depreende-se que as ferramentas usadas nessas aplicações foram os tradicionais recursos de projeto da Bentley,

"Different titles and acronyms have been tested to describe a model-based approach to design for civil engineering - virtual design and construction (VDC) and civil information management (CIM), to name two. However, it is clear that despite early resistance to the term as applied to civil engineering and related disciplines, BIM for Infrastructure is entering the mainstream."

"The project teams for each share a common objective: to manage complex infrastructure projects in an urban environment in the most efficient way. The visions behind the strategies developed by these different organizations are based on the same principle: use an intelligent 3D model to support collaboration across the lifecycle of infrastructure assets, including their planning, design, engineering, construction, and management on into the operations phase." 
integrados e associados a massas de informações semânticas armazenadas em bancos de dados (Oracle) de grande capacidade que suportam os objetos 3D e o formato de arquivo gráfico 3D DGN.

Ainda segundo Bentley (2011), nas lições aprendidas é destacado que a implementação de um $3 D$ City Model para atender às necessidades de infraestrutura colocou essas cidades à frente de muitas outras de mesma categoria, constituindo num processo estratégico que oferece um bom retorno sobre o investimento e que os benefícios mais valiosos são atingidos quando se vai além da visualização tridimensional e contempla um modelo semanticamente rico.

Observa ainda (BENTLEY, 2011) que frequentemente os dados 3D provenientes de CAD ou GIS são contrapostos aos dados 2D usados até recentemente. As aplicações demonstraram que não importa a discussão sobre o uso de dados 2D ou 3D, e sim como fazer a integração mais eficiente possível entre eles. Entretanto, o projeto londrino demonstra claramente como tirar partido das representações 2D extraídas dos modelos 3D. Finalmente, a análise conclui que a modelagem das feições existentes acima e abaixo do solo é essencial para um 3D City Model completo, mesmo porque uma significativa parte da infraestrutura da cidade é subterrânea.

Da leitura da publicação (BENTLEY, 2011) depreende-se que até aquele momento a empresa ainda não tornara pública uma estratégia para o desenvolvimento de uma plataforma CIM, com as características semelhantes ao BIM, mas sim enfrentava o desenvolvimento de aplicações complexas, colaborativas e integradas com os seus recursos (CAD/GIS/CAFM) disponíveis e que a sua visão de CIM se refere à projetação, à construção e à operação da infraestrutura física urbana.

Assim, o esforço da empresa está em assegurar a integração do grande número de ferramentas já disponíveis para as áreas de arquitetura, engenharia, construção e operação de todos os tipos de infraestrutura, incluindo sistemas de serviços públicos, estradas e ferrovias, pontes, edifícios, redes de comunicação, redes de água e de esgotos, dentre outros. Segundo Thein ${ }^{9}$ (2011), na condição de membro fundador da International Alliance for Interoperability (IAI), a Bentley fornece significativos recursos para a definição do formato de dados IFC e trabalha no desenvolvimento de uma interface IFC para as suas aplicações.

\section{Esri}

A visão da Esri expressa num white paper de 2014 (ESRI, 2014) reflete que os 3D urban maps (mapas urbanos 3D) e os modelos de edificações a eles associados estão permitindo a convergência de várias disciplinas estabelecidas, incluindo os desenhos de engenharia feitos no computador (CAD) (sic), o gerenciamento das informações de edificações (BIM) (sic) e sistemas de informações geográficas (GIS).

Um dos primeiros usos comuns de mapeamento urbano 3D é a simulação para modelagem de inundações, que tem crescido em importância desde o furacão Katrina, em 2005. Quando os modelos de edificações são combinados com dados de terreno precisos (DTM) as simulações podem ser criadas para identificar com precisão as áreas de risco com base em uma variedade de cenários hipotéticos (ESRI, 2014) ${ }^{10}$.

Desse modo, um único “mapa urbano 3D” pode conter as informações detalhadas das edificações, representações das características físicas e

\footnotetext{
Volker Thein, IFC Product Manager.

10 "One of the common early uses of 3D urban mapping is simulation for flood modeling, which has grown in importance since Hurricane Katrina in 2005. When building models are combined with accurate terrain data (DTM), simulations can be created that accurately identify areas of risk based on a variety of what-if scenarios."
} 
funcionais das instalações, tudo vinculado a uma localização geográfica tridimensional.

Os mapas urbanos tridimensionais assim compostos, se totalmente modelado com seus atributos, proporcionam um alto grau de compreensão de todo o ambiente urbano e amplia a capacidade de planejar e gerenciar os eventos, possibilitando maior segurança na tomada de decisões. Entretanto, mesmo que parcialmente completos, os mapas tridimensionais dotados de atributos-chave permitem avanços significativos em relação às tradicionais aplicações desenvolvidas em 2D.

No white paper (ESRI, 2014) são citados usos potenciais dos 3D urban maps e são apresentados dois estudos de casos de aplicações no estado do Hawaii e na cidade de Portland nos Estados Unidos.

São exemplos que contemplam a espacialização de leis urbanas, especialmente no que se refere ao zoneamento, seja para a análise da conformidade das edificações, seja para a visualização de cenários futuros em caso de alterações introduzidas nas leis, a realização de estudos solares, como a análise do sombreamento provocado pelas edificações ou ainda, a elaboração de mapas das superfícies com potencial de captação da energia solar para instalação de dispositivos fotovoltaicos. E finalmente, o cálculo do potencial de captação de água de chuva por meio do telhado das edificações a partir da intensidade pluviométrica.

Como esperado, a Esri, tradicional softhouse na área de geotecnologias, foca a sua visão de City Information Modeling numa abordagem de espacialização e análise de informações geográficas, típicas dos GIS, passando ao largo das aplicações voltadas para projetos ou de aspectos da construção dos sistemas de infraestrutura e das edificações.

Entretanto, surpreende quando não faz maiores considerações sobre uma aproximação com o BIM, com a integração com o padrão IFC, ou deixa de abordar a interoperabilidade entre diferentes plataformas ou aplicações. Limita-se a expressar a compatibilidade dos seus formatos de dados com o padrão internacional CityGML e citar o desenvolvimento de um padrão proprietário, o 3D-CIM ${ }^{11}$ para uso em 3D urban mapping.

\section{CAMINHANDO EM DIREÇÃO A UMA CONCEITUAÇÃO}

Considerando-se uma visão sistêmica da cidade, os vários tipos de edificações e os sistemas físicos de infraestrutura urbana são elementos essenciais e estão presentes em qualquer definição de cidade, sendo estes uma condição necessária ao estabelecimento de qualquer assentamento humano.

Nesse sentido, uma visão de CIM tem que contemplar necessariamente uma visão de BIM. Em outras palavras, o conceito de BIM está contido no conceito de CIM, da mesma forma que o conceito de CAD está contido no conceito de BIM. É importante destacar que o fato de estar contido não significa estar superado, ultrapassado ou ainda ser substituído, ser substituível ou mesmo ser de importância menor. A condição "está contido" significa apenas a submissão hierárquica de um sistema em relação ao outro, a partir de uma visão sistêmica, estratégica e integrada.

Assim, mantida a analogia com o BIM enfatizada por diversos autores, um dos principais aspectos a serem definidos é a questão do ciclo de vida no contexto CIM. Como este deve e pode ser caracterizado tanto para a cidade quanto para cada um dos seus complexos subsistemas, mesmo que sejam dotados de características e regulações próprias e administrados por diferentes autoridades.

Um segundo aspecto essencial é a questão do modelo CIM, ou seja, a base de dados que viabiliza as aplicações que atuam sobre o sistema. As informações devem estar centralizadas num modelo único e serão

Estranhamente, a Esri (2014) adota a terminologia City Information Model (3D-CIM) para o seu formato de dados proprietário. Isso de forma alguma contribui para um melhor esclarecimento do conceito ou de uma visão de integração de tecnologias a aplicações. 
acessadas e transacionadas entre os vários subsistemas do CIM, e seus administradores. Entretanto, aqui reside um dos maiores problemas a serem enfrentados, que é a integração dos vários subsistemas e a garantia da interoperabilidade entre eles.

Tradicionalmente, as abordagens sobre a cidade têm sido tratadas em plataformas GIS numa visão temática cobrindo todo o espaço urbano, em aplicações como planejamento urbano, sistemas de abastecimento de água, distribuição de energia elétrica, entre outros, e num recorte também temático, mas com intervenções mais pontuais, por meio de plataformas CAD em aplicações como o projeto de um trecho do sistema viário ou a expansão de uma rede coletora de esgotos ou de toda a infraestrutura de um novo "bairro".

Verifica-se que tanto as plataformas CAD quanto as plataformas GIS apresentam problemas de interoperabilidade no seu próprio escopo (GIS GIS) ou (CAD-CAD) entre os produtos dos diversos fabricantes e mesmo entre os produtos de um mesmo fornecedor. Ou seja, dificilmente as conversões de dados atualmente feitas entre as diferentes plataformas e aplicações acontecem sem a perda de informações e retrabalho. E isto implica em problemas operacionais, tecnológicos e, sobretudo, na necessidade de tempo e custos adicionais.

A implementação de uma plataforma CIM significa que as ferramentas deverão ter características CAD e GIS (GIL et al., 2010) e vai necessitar da definição de um formado de dados que possibilite a integração e a desejada interoperabilidade, como foi pensado o IFC para o BIM.

Diante disto, será o padrão IFC (Industry Foundation Classes) capaz de suportar ou se adaptar para atender às necessidades do CIM? Ou será necessária a criação de mais um padrão para fazer parte do complexo quadro já existente?

Por outro lado, o padrão CityGML (City Geography Markup Language) embora bem definido e documentado, e ainda com uma excelente perspectiva de adoção em escala internacional, inclusive com importantes aplicações implementadas, é semanticamente mais pobre do que o formato IFC, sendo portanto incapaz de suprir as demandas de um CIM.

Assim, de forma análoga ao BIM, a etapa de uso (operação e manutenção) do sistema deverá ter um papel preponderante, demandando especial cuidado na modelagem do CIM, dada a multiplicidade de aspectos envolvidos, de forma que as aplicações de uma SmartCity possam atuar sobre o CIM (trocar informações).

A aplicação SmartCity (sistemas) seria mais voltada para os usuários finais de todos os sistemas urbanos, basicamente constituída pelos cidadãos e administradores, enquanto que a aplicação CIM (sistemas) seria mais votada para aqueles que lidam com a produção e a operação dos sistemas físicos, como planejadores, projetistas, construtores, operadores e administradores.

A seguir, a Figura 1 busca aproximar essa ideia de integração entre os conceitos de CIM e de SmartCity na configuração de uma cidade virtual, que seria a reunião de duas grandes aplicações ou de dois grandes sistema urbanos digitais e as suas respectivas bases de dados.

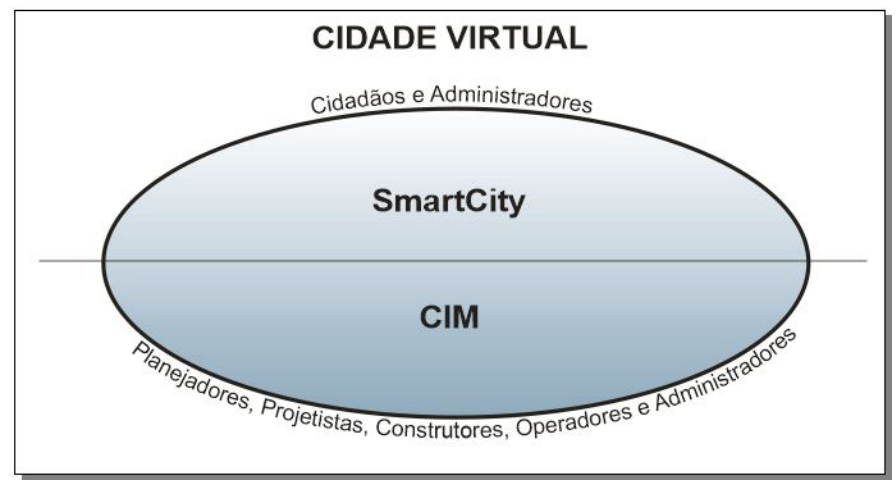

Figura 1. Cidade Virtual: integração CIM / SmartCity. Fonte: Elaborada pelo autor 


\section{CONSIDERAÇÕES FINAIS}

Este trabalho aborda o paradigma City Information Modeling por meio de uma analogia com o Building Information Modeling, da conceituação de outros autores e da análise de outros conceitos correlatos, considerando a visão dos provedores de tecnologia e as ferramentas disponíveis para a implementação de uma plataforma de software dotada das características necessárias.

Não tem a pretensão de esgotar o tema, seja pela vastidão das questões envolvidas, seja também pelo reduzido espaço para a discussão das mesmas, mas principalmente porque essas questões ainda estão "em aberto", sendo objeto de discussões e controvérsias. Visa, portanto, contribuir para uma discussão mais ampla e integrada, e ressaltar a necessidade do encaminhamento das questões envolvidas.

A implantação de um City Information Modeling pode ser um dos caminhos para um cidade alcançar o status de CitySmart, uma vez que o CIM, em última instância, busca a eficácia global de todos os sistemas de infraestrutura urbana, e consequentemente, dos serviços e atividades dependentes desses sistemas de infraestrutura. Já uma SmartCity se caracteriza pelo uso de sistemas e tecnologias de informação e comunicação (TIC), além daqueles contidos no CIM, para atingir a eficiência e a eficácia de todos os sistemas urbanos em benefício da qualidade de vida dos seus cidadãos.

A implantação de um CIM não é suficiente para se chegar à SmartCity. São dois empreendimentos de grande envergadura, tanto pelos custos e riscos tecnológicos envolvidos, quanto pelas dificuldades operacionais, administrativas e legais. Entretanto, eles são dotados de uma relativa interdependência, de uma grande afinidade e capazes de produzir sinergia. A decisão de uma implementação integrada entre City Information Modeling (CIM) e SmartCity reduz os riscos inerentes aos processos, minimiza os custos e maximiza os resultados. De toda sorte, existe um árduo caminho a ser trilhado até a cidade virtual.

Por fim, o que garante de fato o status de uma SmartCity ou de uma Cidade Virtual não é a simples existência, ou mesmo o bom desempenho dos sistemas informatizados de produção e de controle dos vários sistemas urbanos, mas sim o bom desempenho desses "sistemas físicos", comprovados pela satisfação dos seus usuários.

\section{AGRADECIMENTOS}

O autor formaliza o seu agradecimento ao Conselho Nacional de Desenvolvimento Científico e Tecnológico (CNPq) pela bolsa a ele concedida, bem como por financiamento concedido ao grupo de pesquisa LCAD por meio do Edital Universal.

\section{REFERÊNCIAS}

AUTODESK. BIM for Infrastructure: a vehicle for business transformation. Autodesk White Paper, 2012.

BENTLEY. City Information Modeling for Sustaining Cities: lessons learned from advanced users. Bentley: Case Study Showcase, 2011.

BURROUGH, P. A.; MCDONNELL, R. A. Principles of Geographical Information Systems. Oxford: Oxford University Press, 1998.
COWEN, D. J. GIS versus CAD versus DBMS: what are the differences? Photogrammetric Engineering And Remote Sensing, v. 54, n. 11, p. 1551-1555, 1988

ESRI. 3D Urban Mapping: from pretty pictures to 3D GIS. Esri White Paper, dez. 2014.

GIL, J.; ALMEIDA, J.; DUARTE, J. The backbone of a City Information Model (CIM): Implementing a spatial data model for urban design. In: EDUCATION AND RESEARCH 
IN COMPUTER AIDED ARCHITECTURAL DESIGN IN EUROPE, 29., 2011, Ljubljana. Proceedings... Ljubljana, University of Ljubljana / Faculty of Architecture, 2011.

GIL, J.; BEIRÃO, J.; MONTENEGRO, N.; DUARTE, J. Assessing Computational Tools for Urban Design: towards a city information model. In: EDUCATION AND RESEARCH IN COMPUTER AIDED ARCHITECTURAL DESIGN IN EUROPE, 28., 2010, Prague. Proceedings... Prague, Czech Technical University in Prague / Faculty of Architecture, 2010.

GOODCHILD, M. F. Geographical information science: fifteen years later. In: International Journal of Geographical Information Science and Systems. Boca Raton: CRC Press, 2006. p. 199-204. Disponível em: <http://www.geog.ucsb.edu/ good/ papers/424.pdf>. Acesso em: 11 jun. 2011.

GRÖGER, G.; KOLBE, T. H.; NAGEL, C.; HÄFELE, K. H. (Eds.). OGC City Geography Markup Language (CityGML) Encoding Standard. [S. I.]: Open Geospatial Consortium, 2012.

HISHAM, A. The new trend of CIM. In: Ahmad's Findings. Disponível em: <http:// ahmadsfindings.blogspot.de/2010/05/ new-trend-of-cim.html>. Acesso em: 19 abr. 2015.
LEE, J.; HANCOCK, M. Toward a framework

for Smart Cities: a comparison of Seoul, San Francisco \& Amsterdam. Standford Program on Regions of Innovation and Entrepreneurship, 2012.

SELADA, C.; SILVA, C. As cidades inteligentes na agenda europeia: oportunidades para Portugal. In: CONFERÊNCIA DE PLANEAMENTO REGIONAL E URBANO (PRU), 2., ENPLAN, 8., e WORKSHOP APDR, 18., 2013, Aveiro. Anais eletrônicos... Disponível em: <http:// www.inteli.pt/uploads/documentos/ documento_1373454640_1255.pdf>. Acesso em: 22 abr. 2015.

STOJANOVSKI, T. City Information Modeling (CIM) and Urbanism: blocks, connections, territories, people and situations. In: Symposium on Simulation for Architecture and Urban Design, 2013, San Diego. Anais eletrônicos... Disponível em: <http://dl.acm. org/citation.cfm?id=2500016>. Acesso em: 22 abr. 2015.

THEIN, V. Industry Foundation Classes (IFC) - BIM interoperability through a vendorindependent file format. Bentley White Paper, set. 2011.

WIKIPEDIA. Smart City. Disponível em: <http://en.wikipedia.org/wiki/Smart_city>. Acesso em: 20 nov. 2014. 